\title{
Early age of sexual debut: a risky experience
}

\section{Margaret Stanley}

\section{Background}

Any discussion on sexual activity by young adolescents incites the expression of deeply held views accompanied frequently by opinions on its prevention, which - although well meant - are often quite unrealistic. However, the age of sexual initiation (the sexual debut) is of substantial interest in the context of public health and social policy.

The UK at the end of the 20th century had the highest rate of teenage births in Western Europe. There is also a continuing upward trend in the rates of sexually transmitted infections (STIs) amongst young people. Genital human papillomavirus (HPV) infection is an extremely common STI. The lifetime risk of acquiring a genital HPV is between $50 \%$ and $80 \%$. The peak prevalence in women is in the 15-19-year-old age group and the acquisition is usually seen soon after the initiation of sexual activity. Genital warts, also caused by HPV (though by 'low-risk', i.e. not cancer-causing, types), represent the commonest viral STI in the UK with almost 84000 cases reported from sexual health clinics in 2006. The peak incidence in women is again in the 15-19-yearold age group, mirroring the average age of onset of sexual activity in the UK. The relationship between the development of genital warts and the age of sexual debut is easy to understand, but the relationship between early sexual activity and the development of cervical cancer, also an HPV-caused disease, is less intuitive. Cervical cancer manifests decades after infection with HPV and it is not immediately obvious why the age at which a woman acquires 'high-risk' (i.e. cancer-causing) HPV should be a risk factor.

\section{Risk factors for cervical carcinogenesis}

Case control studies have identified age at first intercourse and also age at first pregnancy as important risk factors for the process of cervical carcinogenesis. ${ }^{1}$ The question is, are there biological reasons why the peri-menarchal cervix and the cervix in first pregnancy should be vulnerable to highrisk HPV infection and a subsequent neoplastic process? There are three factors that should be considered:

- Epithelial morphology of the squamo-columnar junction at the menarche and in first pregnancy

- Effect of steroid hormones on the immune status of the adolescent at both these times

- Action of steroid hormones on HPV gene expression and viral replication.

Of central importance to this discussion are the changes that occur at the squamo-columnar junction of the cervix. Stratified squamous non-keratinising epithelium of the ecto-cervix meets the mucous secreting columnar epithelium of the endo-cervix at an abrupt point, the squamo-columnar junction. This is a dynamic structure that undergoes changes in late fetal life, at the menarche and during first pregnancy. ${ }^{2}$ At these times the more caudal of

J Fam Plann Reprod Health Care 2009; 35(2): 118-120

Department of Pathology, University of Cambridge, Cambridge, UK

Margaret Stanley, PhD, OBE, Professor of Epithelial Biology

Correspondence to: Professor Margaret Stanley, Department of Pathology, University of Cambridge, Tennis Court Road,

Cambridge CB2 1QP, UK. E-mail: mas1001@cam.ac.uk the original columnar epithelium is partially or completely replaced with squamous epithelium by the physiological process of squamous metaplasia. This is particularly marked in the first pregnancy when the endocervix everts, exposing the previously protected glandular cells to the acidic vaginal milieu resulting in an extensive area of metaplasia. ${ }^{3}$ Metaplasia is classically defined as the replacement of one differentiated cell type with another, but the squamous epithelium generated in metaplasia is not identical to the native epithelium. The progenitor cell of both squamous and glandular cervical epithelia is widely considered to be the reserve cell. The reserve cell has characteristic keratin and adhesion protein expression and in both immature and mature metaplastic epithelium all the reserve cell keratins are expressed, in contrast to native squamous or columnar epithelium, in which only subsets of the reserved keratins are retained. The differences between native and metaplastic epithelium are particularly marked in immature metaplasia, a proliferative but incompletely differentiated epithelium (Figure 1).

\section{HPV infection and replication}

This all becomes relevant when the way HPV infects keratinocytes is considered. The emerging evidence is that viral entry requires micro-abrasion of the epithelial surface, a micro-wound that removes the epithelium but keeps the basement membrane intact. ${ }^{4}$ The virus then appears to bind to the exposed basement membrane and subsequently will bind to the basal keratinocyte. The thinner, more fragile metaplastic epithelium may be more susceptible to the micro-wounding process and thus to HPV infection. The frequency and extent of the metaplastic transformation seen in pregnancy depends primarily on parity, so eversion of the endocervical epithelium onto the ectocervix is much more likely to occur in the first pregnancy than in subsequent gestations and in the first pregnancy there will be sheets of immature and mature metaplastic epithelium. HPV requires a fully differentiated squamous epithelium for virus production and release, so the incomplete differentiation of metaplasia could result in semipermissive (the virus replication cycle gets stuck before the assembly of mature virus, because the differentiated squamous cells in which the virus coat proteins L1 and L2 are made are not present) viral infection, a state known to promote neoplastic transformation by DNA viruses.

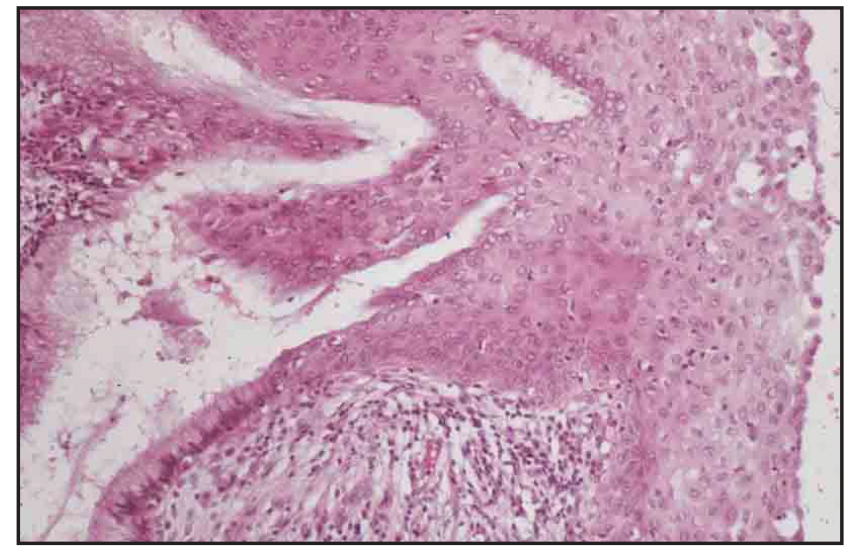

Figure 1 Immature squamous metaplasia. The endocervical epithelium is replaced by proliferative squamous epithelium (photomicrograph author's own) 
Huge changes in the levels of estrogen and progesterone accompany the menarche and pregnancy and these steroids influence epithelial morphology, immune status and HPV replication. Thus reserve cells express estrogen receptors and estrogen-response proteins and the induction of metaplasia has been postulated to have a hormonal basis..$^{5}$ Metaplastic cells have greater numbers of estrogen and progesterone receptors and it has been suggested that the marked immature metaplasia of pregnancy is a consequence of enhanced signalling via these receptors. Estrogens are known to influence immunocompetence and the Th1/Th2 balance in the female genital tract. ${ }^{6}$ Th1 T-cells drive cell-mediated immunity the killing and clearance of virus infected cells; Th2 cells drive humoral or antibody-mediated immunity and prevent re-infection by viruses. Estrogen receptors are expressed by many immunocytes, T-cells, B-cells, dendritic cells, macrophages and natural killer (NK) cells. Importantly, the $\mathrm{T}$ regulatory subset of T-cells (T-regs) undergo profound changes during the ovarian cycle. T-regs are considered to recognise self-antigens and function to prevent autoimmunity, but they also regulate responses to exogenous antigens and have been implicated in chronic viral infections favouring viral persistence. T-regs increase during the follicular phase of the ovarian cycle, when estrogen levels are high, and decrease during the luteal phase. Estrogens also alter expression of chemokine receptors by T-cells; these receptors function as a cellular 'global positioning system' and changes could influence homing to tumour mucosal tissues. Estrogens appear also to exert a biphasic effect on Th1/Th2 differentiation and this seems to be particularly marked in pregnancy where the high estrogen levels of the third trimester favour the generation of Th2 responses. Estrogen effects on late responses are largely repressive for Th1 responses, downregulating receptor expression and the secretion of pro-inflammatory cytokines by macrophages. Furthermore, estrogens decrease the cytotoxicity of NK cells and affect the microvascular endothelial cells that actively recruit immune and inflammatory cells, further enhancing the anti-inflammatory milieu. HPV infections are cleared by Th1-biased cell-mediated cytotoxic immune responses. Although the cytotoxic effectors involved are not well understood, it is clear that under high estrogen blockade the immune milieu would not favour clearance of HPV infections. This is further emphasised by the emerging evidence that implicates activated innate immune cells, particularly NK cells, as important cytotoxic effectors in the clearance of HPV infections. Overall, the hormonal milieu around the menarche and in first pregnancy would lead towards HPV persistence rather than HPV clearance.

\section{Steroid hormones and HPV gene expression}

Finally, steroid hormones can directly influence HPV gene expression. The region of the HPV genome that regulates HPV gene expression contains so-called steroid response elements; estrogens can directly up-regulate the expression of the E6 E7 oncogenes by binding to these elements. There is very strong evidence from HPV16 E6/E7 transgenic mice that estrogens synergise with these oncogenes in both the initiation and progression of cervical cancer. ${ }^{7}$ In these mice HPV16 E6 and E7 are constantly expressed at low levels in the cervical epithelium but only mice chronically treated with $17 \beta$ estradiol undergo the spectrum of squamous metaplasia - intraepithelial neoplasia - invasive cancer. In these models, withdrawal of exogenous estrogen reduces the frequency of cancer, suggesting that either continuous estrogen exposure or increased estrogen levels will enhance the progression from pre-invasive to invasive cancer.

\section{Acquisition of cervical HPV infection}

A plausible scenario that emerges from all these data is as follows. The acquisition of cervical HPV infection is more likely in early adolescence and first pregnancy because of the epithelial instability at the transformation zone and the fragility of immature metaplasia that is more prone to microabrasion and therefore viral entry. The hormonal milieu, particularly the increased estrogen levels around the menarche and in first pregnancy, results in an immune regulatory and anti-inflammatory milieu that favours the persistence of HPV infection rather than clearance. The stage is then set, with a persistent HPV infection in an incompletely differentiated epithelium in a young adolescent at the start of reproductive life with multiple ovarian cycles ahead. If this is a high-risk HPV then a significantly increased probability of progression to high-grade intraepithelial neoplasia and eventually invasive cancer exists. Sex around the menarche is indeed a risky experience.

\section{Cervical cancer screening}

In the UK the incidence of cervical cancer has been significantly reduced by population-based cervical cancer screening and the UK screening programme is regarded, justifiably, as one of the best in the world. However, screening has had relatively little impact on cervix cancer incidence in women under 30 years of age and this statistic was the rationale for raising the age at which women are first called for screening in England from 20 to 25 years, ${ }^{8}$ though opinion remains divided on the issue. ${ }^{9-11}$ The epidemiological evidence does implicate early sexual debut and early pregnancy as risk factors and the biological rationale for this is given above. Have the epidemiological studies been able to take into account the lowering age at sexual debut apparent in the last $10-15$ years ${ }^{12}$ or will we only see the effects of this risk factor in a few years' time? Meanwhile, it can still be argued that screening of this atrisk population younger than 25 years is justified.

\section{Statements on funding and competing interests}

Funding None identified.

Competing interests The author is a consultant for Merck Vaccines, GlaxoSmithKline Biologicals and Sanofi Pasteur MSD.

\section{References}

1 Green J, Berrington de Gonzalez A, Sweetland S, Beral V, Chilvers C, Crossley B, et al. Risk factors for adenocarcinoma and squamous cell carcinoma of the cervix in women aged 20-44 years: the UK National Case-Control Study of Cervical Cancer. Br J Cancer 2003; 89: 2078-2086.

2 Singer A, Jordan JA. The functional anatomy of the cervix, the cervical epithelium and the stroma. In: Jordan JA, Singer A (eds), The Cervix (2nd edn). Oxford. UK: Blackwell Publishing Ltd, 2006; 13-37.

3 Singer A. The cervical epithelium and subepithelium during pregnancy and the puerperium. In: Jordan JA, Singer A (eds), The Cervix (2nd edn). Oxford, UK: Blackwell Publishing Ltd, 2006; 102-121.

4 Roberts JN, Buck CB, Thompson CD, Kines R, Bernardo M, Choyke PL, et al. Genital transmission of HPV in a mouse model is potentiated by nonoxynol-9 and inhibited by carrageenan. Nat Med 2007; 13: 857-861.

5 Remoue F, Jacobs N, Miot V, Boniver J, Delvenne P. High intraepithelial expression of estrogen and progesterone receptors in the transformation zone of the uterine cervix. $A m \mathrm{~J}$ Obstet Gynecol 2003; 189: 1660-1665.

6 Fish EN. The X-files in immunity: sex-based differences predispose immune responses. Nat Rev Immunol 2008; 8: 737-744.

7 Brake T, Lambert PF. Estrogen contributes to the onset, persistence, and malignant progression of cervical cancer in a human papillomavirus-transgenic mouse model. Proc Natl Acad Sci USA 2005; 102: 2490-2495. 
8 Sasieni P, Adams J, Cuzick J. Benefit of cervical screening at different ages: evidence from the UK audit of screening histories. Br J Cancer 2003; 89: 88-93.

9 Sasieni P, Castanon A, Parkin DM. How many cervical cancers are prevented by treatment of screen-detected disease in young women? Int J Cancer 2009; 124: 461-464.

10 Sigurdsson K, Sigvaldason H. Is it rational to start populationbased cervical cancer screening at or soon after age 20 ?
Analysis of time trends in pre-invasive and invasive diseases. Eur J Cancer 2007; 43: 769-774.

11 Herbert A, Holdsworth G, Kubba A. Cervical screening: why young women should be encouraged to be screened. J Fam Plann Reprod Health Care 2008; 34: 21-25.

12 Wellings K, Nanchahal K, Macdowall W, McManus S, Erens B, Mercer $\mathrm{CH}$, et al. Sexual behaviour in Britain: early heterosexual experience. Lancet 2001; 358: 1843-1850.

\section{What a load of rankers}

\section{Sue Perankin}

So very soon anyone who can access the Internet will be able to leave a defamatory remark about me, thanks to the latest stage of the Government's ongoing GP-bashing campaign, or "we're out to get you" as I like to call it. Under the guise of choice (how I hate that word), patients will be able to log on to the NHS Choices website and say what they think of GPs like me. Some choice, because once it's up there it's going to be impossible to get rid of it, or for me to explain the truth which is, for example: "No, the reason why I refused to give you a pregnancy test was because you've had a hysterectomy, and they removed your ovaries, and you told me you'd not even been in the same room as a man for 5 years, and because I had a bloody good look out of the window and there was no bright light, and neither were there any shepherds or wise men in the waiting room". What a nightmare.

Why do we need this? We don't, is the short, and honest, answer. Survey after survey reports how good we are in our patients' eyes. And therein lays the nub. How can the Government's agenda to bash GPs be fulfilled if the public keeps on saying we're so wonderful? Anyone who bashes GPs shoots themselves in the foot because doing so backfires on them. "How dare you say that about my wonderful doctor, you nasty politician you. I'll not vote for you again." So how do you turn people? You infiltrate, or start a smear campaign.

This whim provides an opportunity for free speech the choice to say something - whilst driving traffic through the NHS website. It's a perfect plan for our taskmasters' aims. Moreover, it's foolproof because it's so one-sided. Rules of patient confidentiality prevent us from giving the facts and defending ourselves, from giving our side of the story. For instance being able to say: "Actually, I didn't call her a slag. What I said was that she appears to be coming in to see me on a monthly basis wanting to be tested for sexually transmitted diseases, so perhaps she should think about using condoms in the future". Then there are the more difficult and career-threatening misinterpretations because of language or accent: "I would never use that word. What I was trying to explain was that under the circumstances there are things that need to be done you can't face".

It's so perverse, and not in a "Oh, that feels so good. I've always wanted to try this" kind of way. Comments posted on the website could sully my reputation, leaving me viewed as an uncaring bastard, it's true. But the knock-

J Fam Plann Reprod Health Care 2009; 35(2): 120

Chartswell Surgery, Bloughinham, UK

Sue Perankin, MRCGP, General Practitioner on effect of this will be the undermining of a patient's confidence in me, a GP who in their eyes had always done very well by them as practice surveys have confirmed annually. In turn the burden of additional stress will make the previously well and happy patient sick, and the unwell patient sicker. End result: a Government plan that makes people sick and adds further pressure on an already drowning NHS. Brilliant.

I'm trying to see a positive to this latest wheeze that isn't more than our paymasters feebly trying to find something to justify not giving the NHS IT system a oneway ticket to Switzerland. Logically you'd think that if a patient believed their GP was dreadful, they'd change GP. But they don't, do they? They stay and make our lives even more miserable. Conversely, I could be swamped with eager gift-bearing patients who want to be looked after by the "so caring and considerate" doctor listed on the site, so every cloud and all that.

I can always dream but why are we surprised when we get a thank you from our patients, because we're more used to getting a complaint. Go to a group or public meeting and what happens? The people with an axe to grind are those who come along. Set up a support group and it's moaners who join. Set up a facility to rank and leave comments about your GP and you don't need me to tell you who are most likely people to leave a comment and what the tone of that comment might be.

Oh heck, I need something to ensure I get a good NHS Choices rating - after all, I am a star. I think I have just the ticket. It's time to return to the old days, the Dr Kildare days, that many people think is current practice, because general practice is still portrayed like this in modern medical TV dramas. That means going back to unnecessary home visits to drop off information leaflets and to deliver appointment details in person, cold phone calling just to "make sure you are OK", and the true art of medicine, which of course is all tea, sympathy and bluff.

Nowadays you can't assume it's only young people who'll use this facility because the older population use the Internet too. I guess I'll need to convince my patients that the Internet is a nasty sex-promoting thing where anyone can say whatever they like, and that you shouldn't believe what's on it, so their stock response becomes: "Oh, I don't believe what they say about you. You've always been wonderful to me". To which I'll reply: "I expect you'll be needing another prescription for lorazepam then? And, perhaps you'd like to write what you just said here in this box on the screen for me? Thank you, that's perfect. What's it all about? Oh, it's just some new thing they want us to do, you know how it is, about ranking us. So I want to make sure I have a good rank."

"That's disgraceful, doctor." "Yes, it is, isn't it?" 